\title{
Helical cutting as a new method for tendon-lengthening in continuity
}

\author{
Farshad, M ; Gerber, C ; Snedeker, J G ; Meyer, D C
}

\begin{abstract}
Helical cutting reliably leaves the tendon in continuity, allows more lengthening than that which can be achieved with z-plasty, and can offer improved resistance to tensile loads.
\end{abstract}

DOI: https://doi.org/10.2106/JBJS.J.00246

Posted at the Zurich Open Repository and Archive, University of Zurich ZORA URL: https://doi.org/10.5167/uzh-53999

Journal Article

Published Version

Originally published at:

Farshad, M; Gerber, C; Snedeker, J G; Meyer, D C (2011). Helical cutting as a new method for tendonlengthening in continuity. Journal of Bone and Joint Surgery. American volume, 93(8):733-738.

DOI: https://doi.org/10.2106/JBJS.J.00246 
The Journal of Bone E Joint Surgery
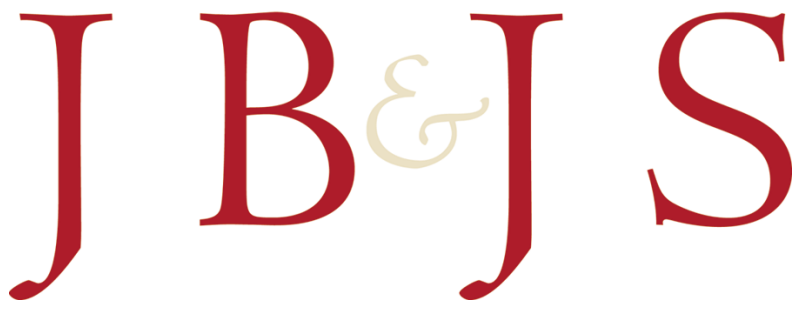

This is an enhanced PDF from The Journal of Bone and Joint Surgery

The PDF of the article you requested follows this cover page.

\section{Helical Cutting as a New Method for Tendon-Lengthening in Continuity}

Mazda Farshad, Christian Gerber, Jess G. Snedeker and Dominik C. Meyer

J Bone Joint Surg Am. 2011;93:733-738. doi:10.2106/JBJS.J.00246

This information is current as of April 22, 2011

Reprints and Permissions

Click here to order reprints or request permission to use material from this article, or locate the article citation on jbjs.org and click on the [Reprints and Permissions] link.

Publisher Information

The Journal of Bone and Joint Surgery

20 Pickering Street, Needham, MA 02492-3157

www.jbjs.org 


\title{
Helical Cutting as a New Method for Tendon-Lengthening in Continuity
}

\author{
By Mazda Farshad, MD, Christian Gerber, MD, FRCSEd(Hon), Jess G. Snedeker, PhD, and Dominik C. Meyer, MD \\ Investigation performed at the Department of Orthopaedics, University Hospital Balgrist, Zürich, Switzerland
}

\begin{abstract}
Background: Additional tendon length is occasionally needed for the surgical reattachment of retracted tendons and for lengthening of intact contracted tendons. To achieve additional length with use of established techniques such as z-plasty, the tendon is completely transected and loses continuity. The purpose of this study was to develop a new method to increase the potential range of attainable tendon length while preserving a degree of tendon continuity.
\end{abstract}

Methods: Forty Achilles tendons (thirty-five study tendons and five reference tendons) were harvested from freshly slaughtered calves. Thirty of the forty tendons were cut along a helical axis located at the tendon centerline, with helical angles of $60^{\circ}, 45^{\circ}$, and $30^{\circ}$; these tendons either were left unsutured or were sutured with mattress stitches along the cut lines. To provide a performance benchmark, five of the forty tendons were lengthened with use of conventional z-plasty. Five more of the forty tendons were left untreated to serve as a general point of reference. Failure behavior was quantitatively assessed in uniaxial tension.

Results: Standard z-plasty yielded a mean length increase (and standard deviation) to $172 \% \pm 10 \%$ of the original length, with a mean tensile strength of $70 \pm 15 \mathrm{~N}$. With use of helical cutting, a wide range of lengths and strengths were achieved, depending on the helical angle. A maximal length increase $(279 \% \pm 80 \%$ of the original length $)$ was achieved with a cut angle of $30^{\circ}$, with an associated load to failure of $30 \pm 7.6 \mathrm{~N}$. In tendons cut helically with an angle of $60^{\circ}$, a length of $212 \% \pm 29 \%$ was achieved, with a corresponding load to failure of $222 \pm 62 \mathrm{~N}$.

Conclusions: Helical cutting reliably leaves the tendon in continuity, allows more lengthening than that which can be achieved with z-plasty, and can offer improved resistance to tensile loads.

Clinical Relevance: Compared with the existing approaches to tendon-lengthening, helical cutting of tendons offers the potential for increased tendon length and better biomechanical performance. This in vitro study lays a biomechanical foundation for a subsequent clinical investigation in patients.

A dditional tendon length is occasionally needed for the surgical reattachment of retracted tendons or for the lengthening of contracted musculotendinous units. Different techniques have been developed, such as $\mathrm{z}$-plasty ${ }^{1,2}$, triple hemisection ${ }^{3}$, the Vulpius technique ${ }^{4}$, the Baker technique $^{5}$, the White slide elongation technique ${ }^{6}$, the so-called piecrust technique used in capsular release ${ }^{7}$, and other variants of these methods. With use of these techniques, the continuity of the tendon is first disrupted and then subsequently repaired to achieve additional length. In existing methods, the final length of the tendon requires a certain degree of planning, is limited in magnitude, and mostly requires suture repair to restore continuity after transection. Furthermore, the immediate postoperative tensile strength of the lengthened tendons is mainly reliant on the suture.

The purpose of this study was to develop a new method of tendon-lengthening in which continuity is preserved and a high degree of additional length is achievable. The first hypothesis was that tendon-lengthening by helical cutting (Fig. 1) will permit additional length, as compared with that permitted by traditional methods, while preserving inherent

Disclosure: The authors did not receive any outside funding or grants in support of their research for or preparation of this work. Neither they nor a member of their immediate families received payments or other benefits or a commitment or agreement to provide such benefits from a commercial entity. 
The Journal of Bone \& Joint Surgery $\cdot$ Jbjs.org Volume 93-A • Number $8 \cdot$ A A Ril 20, 2011

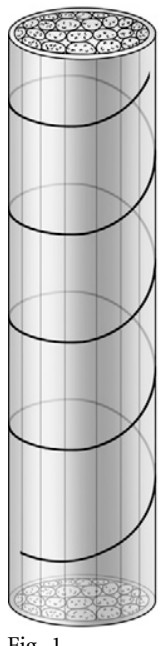

Fig. 1

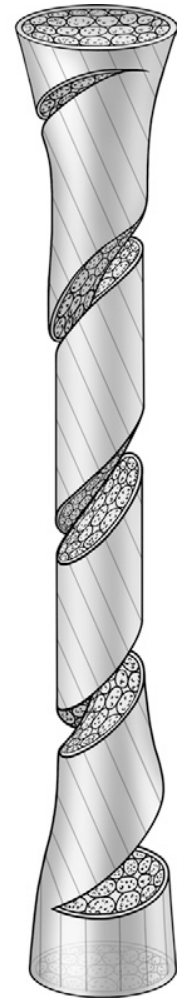

Schematic illustration of the helical cutting technique that allows tendon-lengthening without complete disruption of tendon continuity. The original length of the tendon (left) can be increased (right) by the helical cut.

tensile strength even without suture repair. The second hypothesis was that the length and tensile strength of the final construct can be predicted and controlled by the choice of the helical cut angle. The third hypothesis was that the new method is biomechanically comparable or superior to the widely used z-plasty procedure for tendon-lengthening.

\section{Materials and Methods}

The study was designed as an in vitro experiment on Achilles tendons of freshly slaughtered calves. A mathematical formula was developed to better understand and eventually predict tendon-length gain as a geometric function of the cutting angle in degrees $(\varnothing)$, the preoperative tendon radius (a) and the preoperative tendon length $\left(\mathrm{L}_{\text {Init }}\right)$ (Fig. 2).

The resulting tendon length was assumed to be equivalent to the extended length of the outer helical cut line. The resulting length depends in part on the pitch length (p) of the helical cut (the axial distance between successive turns), which can be approximated with use of the following equation $^{8}$ :

$$
p=4^{*} \tan \left(\frac{\phi}{57.3}\right) * a
$$

Thus, the number of turns $(\mathrm{N})$ required to span the targeted axial length of the cut can be expressed as follows:

$$
N=\frac{L_{\text {Init }}}{p}
$$

Helical Cutting as a New Method for

TENDON-LENGTHENING IN CONTINUITY

And the resulting length of the outer helical cut line can then be described as follows:

$$
L_{\text {Final }}=N^{*} 2 \pi \sqrt{a^{2}+\left(\frac{p}{2 \pi}\right)^{2}}
$$

The relative change in length can thus be described as follows:

$$
\frac{L_{\text {Final }}}{L_{\text {Init }}}=\frac{2 \pi}{p} \sqrt{a^{2}+\left(\frac{p}{2 \pi}\right)^{2}}
$$

The formulas were used to predict resulting additional length that could be achieved in tendons according to cutting angles of $30^{\circ}, 45^{\circ}$, and $60^{\circ}$, assuming an axial cut length of $5 \mathrm{~cm}$ and a tendon diameter of $1 \mathrm{~cm}$. Based on the lowest expected amount of lengthening (to $135 \%$ of original length) with a cut angle of $60^{\circ}$ and assuming a standard deviation of $20 \%$ on the basis of preliminary experiments, power analysis indicated that five tendons would be sufficient to achieve significance, defined by $\mathrm{p}<0.05$.

\section{Surgical Technique and Group Design}

Forty Achilles tendons (thirty-five study tendons and five reference tendons) were immediately harvested from freshly slaughtered calves and carefully dissected from the surrounding connective tissues. Isolated round portions of the tendons were wrapped in saline-solution-soaked gauze and stored at $4^{\circ} \mathrm{C}$ until the time of lengthening and eventual mechanical testing. The central portion of the tendon, excised $2 \mathrm{~cm}$ from its muscular origin and $2 \mathrm{~cm}$ from its osseous insertion site, with an approximately circular cross section, was used for these investigations. The mean diameter of the tendons was $1.10 \pm 0.13 \mathrm{~cm}$. Thirtyfive of the forty tendons, in groups of five tendons each, were randomly assigned to each testing condition, while five of the forty tendons were left intact (untreated) to serve as a general point of reference.

In all tendons, a length of $5 \mathrm{~cm}$ was predesignated as the cutting zone. To define the helical axis along the tendon midline, a hollow needle was first inserted approximately along the longitudinal central axis of the tendon. The hollow needle was used to guide the insertion of a size-0 PDS (polydioxanone) suture (Ethicon, Norderstedt, Germany). The needle was subsequently removed. A spiral cut was performed in the cutting zone to the depth of the

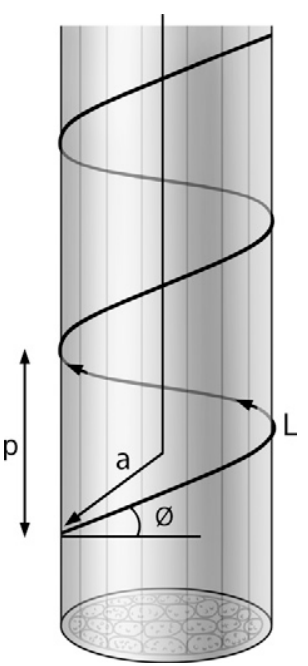

Fig. 2

Illustration of the geometric basis used to predict a theoretical gain in length as a function of the cutting angle in degrees $(\varnothing)$, the preoperative tendon radius (a), and the preoperative tendon length $(L) . p=$ pitch length. 
The Journal of Bone \& Joint Surgery · Jbjs.org Volume 93-A · Number 8 · April 20, 2011
Helical Cutting as a New Method for

TENDON-LENGTHENING IN CONTINUITY suture, approximately corresponding to the tendon midline, with a regular number-10 scalpel blade. A template was used to achieve the intended cutting angle. Angles of $60^{\circ}$ (groups I and IV), $45^{\circ}$ (groups II and V), and $30^{\circ}$ (groups III and VI) were used. In groups IV, V, and VI, mattress sutures were placed along the cut lines in the following manner: a longitudinal pretension of $5 \mathrm{~N}$ was applied to the tendon while suturing with a size-0 PDS suture tightly enough to achieve contact of the cut edges. The sutures were spaced approximately $0.75 \mathrm{~cm}$ apart. The mean achieved length of the helically cut and sutured groups was measured and used to define an intended target length of tendons in group VII, in which a z-plasty lengthening was employed. For the z-plasty, a longitudinal cut $(5 \mathrm{~cm}$ in length) was performed, followed by suturing of the 2 to $3-\mathrm{cm}$ overlapped arms with use of two or three mattress sutures (size-0 PDS) spaced approximately $0.75 \mathrm{~cm}$ apart. This resulted in a final length of approximately $7 \mathrm{~cm}$, which is an increase in length that is consistent with current use of the method ${ }^{9-12}$. Additionally, five tendons were left intact (untreated) to serve as a general point of reference. When not being handled, all tendons were wrapped in saline-solution-soaked gauze to prevent tissue dehydration.

\section{Biomechanical Testing}

Each tendon was clamped for testing in a universal material testing machine (Zwick 1456, Zwick, Ulm, Germany) to establish the force-strain curve for load to failure, where tendon strain was defined as a percentage of the nominal tendon length at a pre-load of $1 \mathrm{~N}$ (Fig. 3). Data were analyzed using standard software (testXpert, version 10; Zwick). Ultimate load (Fmax), strain at ultimate load $\left(\varepsilon_{-} \max \right)$, and strain at complete rupture ( $\varepsilon_{-}$rupture) were determined for each tendon. Rupture was defined as inability to withstand a tensile load greater than $10 \mathrm{~N}$.

\section{Statistical Analysis}

Data were statistically analyzed using analysis of variance for intergroup differences. The Spearman correlation was used to relate achieved length increase to the applied cutting angle. All statistical tests were performed with use of a commercial statistical software package (PRISM, version 5.01 for Windows; GraphPad Software, La Jolla, California), with significance levels set at $\mathrm{p}<0.05$.

\section{Source of Funding}

This study was internally financed and no external funding was employed.

\section{Results}

Tendon-lengthening by helical cutting yielded continuous tendons with relatively high tensile strength and a controllable amount of length gain; the highest tensile strength $(222 \pm 62 \mathrm{~N})$ was observed in group IV $\left(60^{\circ}\right.$ helical lengthening with sutured cut lines). These tendons had a mean strain at ultimate load of $212 \% \pm 29 \%$. Strain at complete rupture was $478 \% \pm 42 \%$. The lowest ultimate load was observed in $30^{\circ}$ helically cut tendons without suturing (group III: $30 \pm 7.6 \mathrm{~N}$ ), with an according strain at ultimate load of $279 \% \pm 80 \%$. In that group, the strain at complete rupture was $310 \% \pm 90 \%$. The tendons that were helically cut and sutured (groups IV, $\mathrm{V}$, and VI) uniformly achieved higher ultimate load than did corresponding tendons without suturing (groups I, II, and III) $\mathrm{p}<0.05$ (Fig. 4). As a general point of reference, untreated tendons reached an ultimate load of $1292 \pm 117 \mathrm{~N}$ with an additional strain at ultimate load of 3.6\% $\pm 0.6 \%$.

The length and tensile strength could be partially controlled by choice of helical cut angle; in the groups for which the cut tendons were not sutured, the cut angle was negatively correlated to strain at ultimate load $(\mathrm{r}=-0.66, \mathrm{p}=0.010)$ and
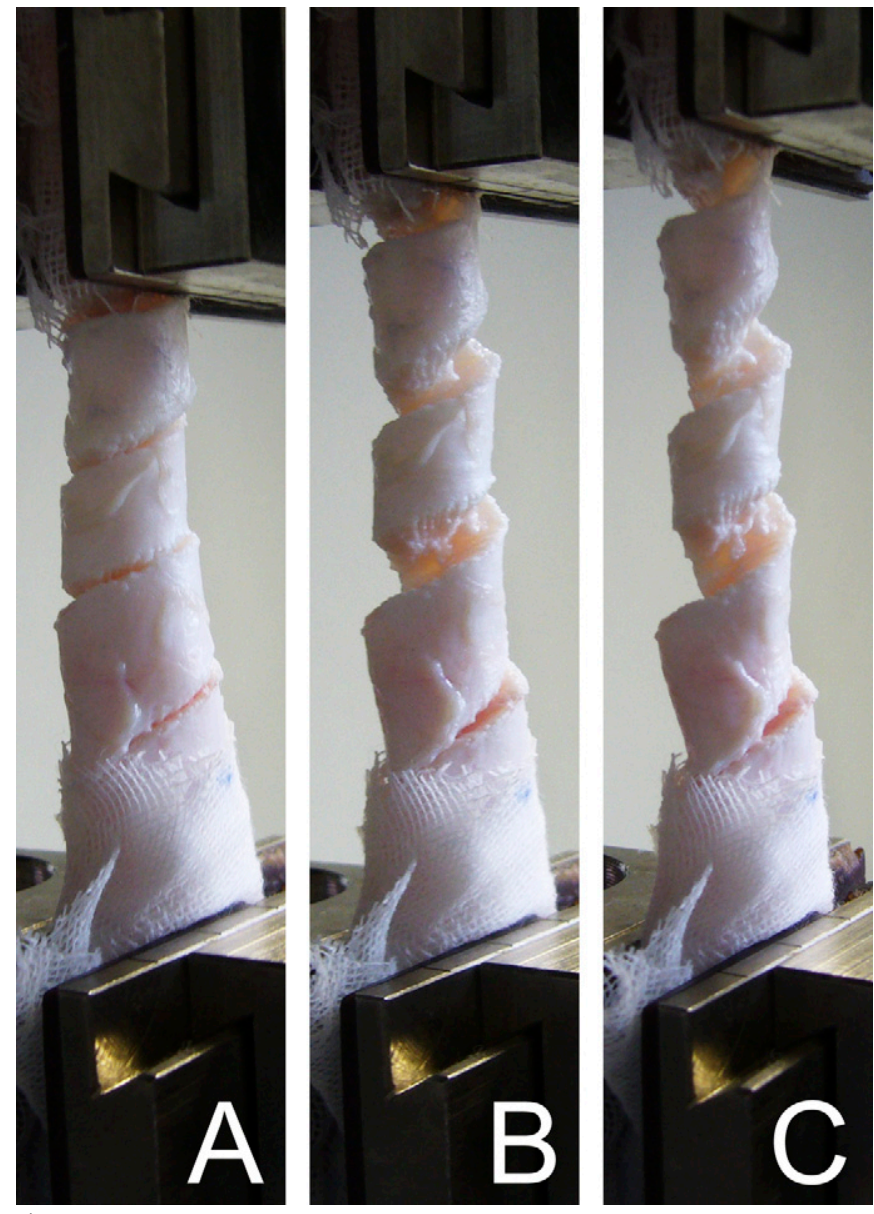

A helically cut tendon with a cut angle of $30^{\circ}$ adapts continuously (from A to $\mathrm{C}$ ) to the tensile load that is applied by the universal material testing machine.

positively correlated to ultimate load $(r=0.72, p=0.003)$. If the helically cut tendons were sutured, there was no correlation between strain at ultimate load and cut angle $(\mathrm{r}=-0.01, \mathrm{p}=$ 0.96 ) but strong positive correlation between ultimate load and cut angle $(\mathrm{r}=0.89, \mathrm{p}<0.0001)$.

The theoretical maximal achievable length (in relation to the original length), calculated with use of the mathematical formula described earlier in the text (and depicted in Fig. 2) was $135 \%, 186 \%$, and $289 \%$ for the $60^{\circ}, 45^{\circ}$, and $30^{\circ}$ cut angles, respectively. The experimentally determined strain at ultimate load of the helically cut tendons (groups I, II, and III) was $167 \% \pm 22 \%, 167 \% \pm 19 \%$, and $279 \% \pm 80 \%$ for the $60^{\circ}$, $45^{\circ}$, and $30^{\circ}$ helical cut angles, respectively.

In group VII (the z-plasty group), a strain at ultimate load of $172 \% \pm 10 \%$ was achieved with a corresponding ultimate load of $70 \pm 15 \mathrm{~N}$. Other than in groups III and VI, where the cut angle was $30^{\circ}$, significantly higher ultimate tensile strengths were observed in helically lengthened tendons (from a minimum of $80 \pm 54 \mathrm{~N}$ in group II to a maximum of $222 \pm 62 \mathrm{~N}$ in group IV). Compared with z-plasty, strain at ultimate load was similar in groups I and II and higher in the other groups (Fig. 4). 
The Journal of Bone \& Joint Surgery $\cdot$ JbJs.org

Volume 93-A - Number 8 - April 20, 2011

Ultimade load (sutured)
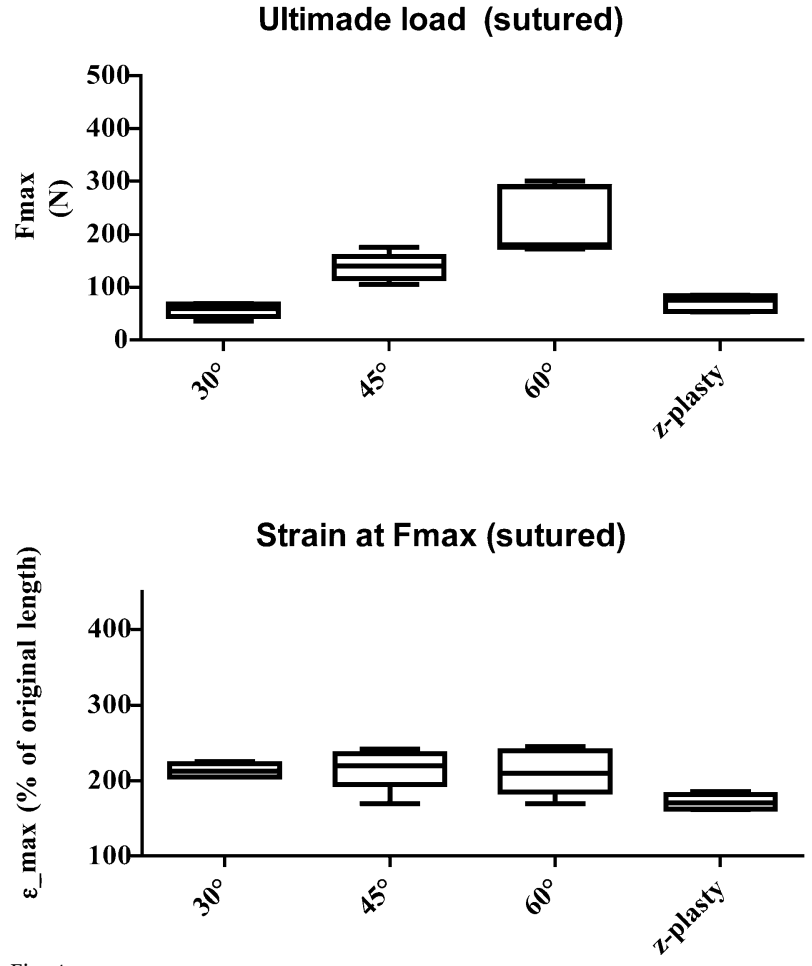

Fig.
Helical Cutting as a New Method for

Tendon-Lengthening in Continuity
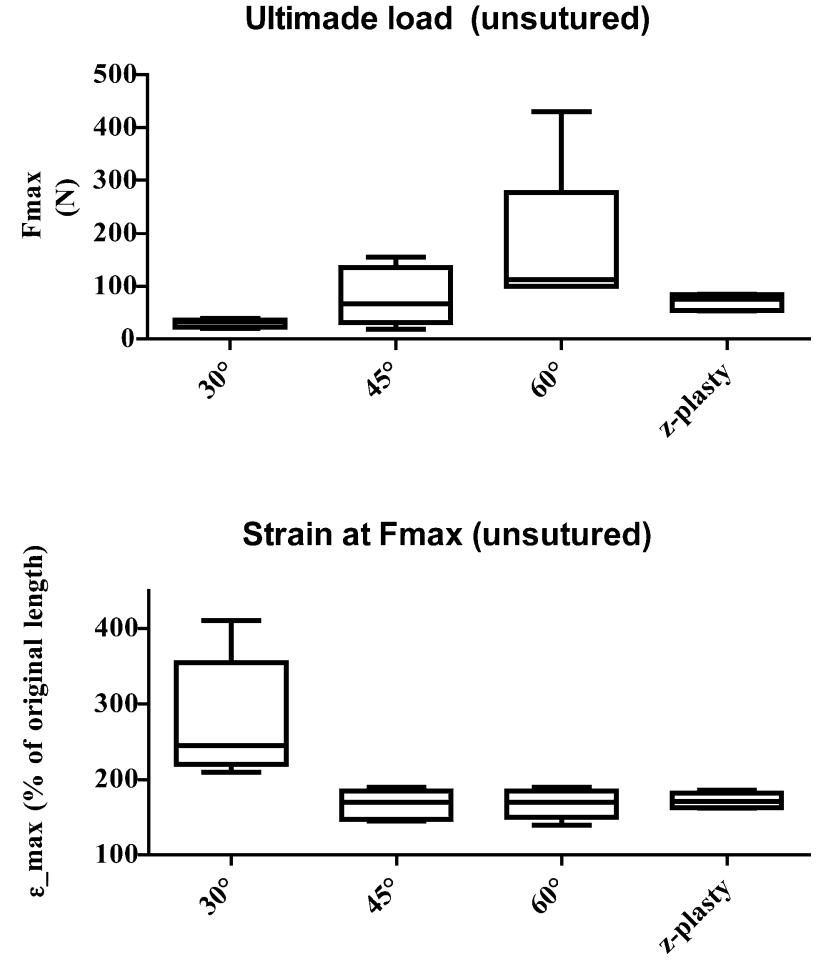

The ultimate load (Fmax) and the strain at this load ( $\left.\varepsilon_{-} \max \right)$ in relation to the original length for each group.

\section{Discussion}

$\mathrm{C}$ urrent clinical techniques for tendon-lengthening, such as z-plasty, completely disrupt the tendon and reconstruct it with sutures to attain additional length. The mechanical integrity of tendon that is fully transected, reconfigured, and then repaired will initially depend on the ability of the sutures to bridge the disrupted collagen bundles until healing has occurred. Until then, the sutured tendon must be protected from overload. After healing, the entire load will inevitably be transmitted through scar tissue. It would therefore be desirable to develop new methods for tendon-lengthening in which tendon continuity is not fully disrupted, a high degree of additional length can be achieved, and some inherent postoperative tensile strength can be maintained. The purpose of this study was to develop such a technique with use of the principle of helical cutting and to investigate whether the amount of additional length could be controlled by selecting an appropriate cut angle. We also sought to characterize the in vitro biomechanical performance of the new technique in comparison with that of $\mathrm{z}$-plasty.

There are limitations to this ex vivo investigation. The healing behavior of helically lengthened tendons in vivo remains unknown and must be explored further in future work. However, the preservation of continuity might be favorable not only with regard to postoperative tensile strength but also with regard to healing because the nutritive paratendon ${ }^{13}$ is not fully disrupted. Furthermore, if left unsutured, helically cut tendons are highly compliant (Fig. 5), which may have implications with regard to the contractile tone of the attached muscle and could potentially permit muscle retraction with adverse con- sequences such as atrophy, fibrosis, fatty infiltration ${ }^{14}$, and loss of muscle strength. However, we have demonstrated that suturing of helically cut tendons can result in more robust tendons that have stiffness similar to that seen in tendons that have been longitudinally cut with z-plasty (Fig. 5). Thus, intraoperative suturing of helically lengthened tendons may be advisable to prevent uncontrolled postoperative tendon-lengthening, even at forces at which traditional z-plasty-lengthened tendons could fail. The effect of different suturing techniques and the tightness of suturing have not been investigated here and are subjects of future research. A second limitation is that the experiments performed here employed tendons with a nearly circular cross section. The feasibility of helical cutting is not yet clear for tendons with an asymmetrical cross section. As a third limitation, the mathematical formula we introduce must be viewed as approximate. The model assumes that the achievable tendon length is equivalent to the length of the cutting line along the perimeter of the tendon. These theoretical values were compared with the experimental values (strain at ultimate load) of fully loaded tendon, and thus the experimental values should be expected to exceed the predicted values. Conversely, the model tends to overpredict the achievable length of highly oblique helical cuts (e.g., $30^{\circ}$ ), which is likely due to the complex load-bearing that occurs in helically cut tendons, as described below.

In helically lengthened tendons, some continuity of the tendon persists to provide additional mechanical strength, but this added strength relies on shear transfer of load between transected collagen fascicles. The tendon tested here (calf Achilles 


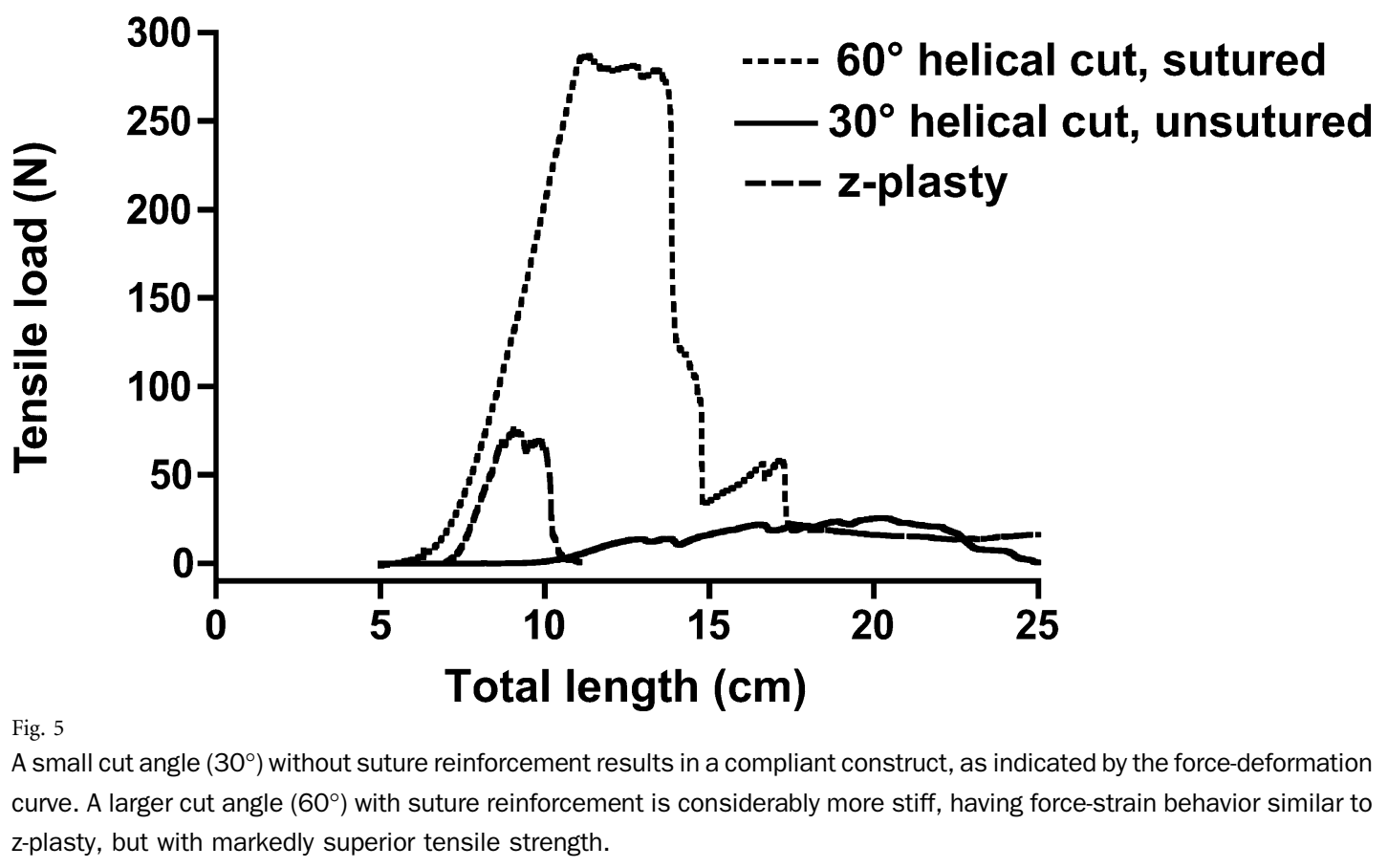

tendon) represents a case in which the lateral connections between neighboring collagen fascicles are fairly robust and the helically cut tendon maintains mechanical strength through effective shear transfer between the transected collagen structures. At more severe cut angles (e.g., $30^{\circ}$ ) the disruptions of the fascicles are more closely spaced, and the shear transfer mechanisms become increasingly critical. Tendons that have less effective shear coupling between neighboring fascicles can be expected to be less robust following helical cutting. At some point, the ability of the shortened tendon structures to bear these shear loads reaches a limit, and the unsutured structure can become unstable. Furthermore, the microarchitecture of tendon tissue is known to vary substantially between different tendons, and this can further differ in degenerate tendon ${ }^{15,16}$. In the calf Achilles tendon, the collagen fascicles are long and arranged nearly parallel to one another. We believe that less homogeneously organized tendons (i.e., tendons with collagen bundles oblique to the central axis) will be more robust after helical cutting, since the effective length of the disrupted segments imposed by the helix will be longer. Thus the inherent mechanical strength of helically cut tendons (without sutures) will vary for specific tendons and various indications, and further investigation is warranted for the target tendon type in each indication.

Testing of our first hypothesis confirmed that, in comparison with tendon-lengthening by z-plasty, tendon-lengthening by helical cutting can add substantial length while improving tensile strength. An ultimate tensile strength (ultimate load or Fmax) of $222 \pm 62 \mathrm{~N}$ was observed in helically cut tendons with a $60^{\circ}$ cut angle and subsequent suturing (group IV) while more than doubling the original tendon length $(212 \% \pm 29 \%$ at peak force). These load limits fall far short of the intact tendon strengths tested here $(1292 \pm 117 \mathrm{~N})$, but exceed strength values reported for repaired human Achilles tendons that range between $120 \mathrm{~N}$ and $150 \mathrm{~N}$, depending on the repair technique that is applied ${ }^{17}$. It can thus be assumed that helical cutting with a $60^{\circ}$ cut angle would still not permit direct postoperative full weight-bearing but could reduce the likelihood of reconstruction failure and possibly reduce the required immobilization time. However, verification of this conjecture is grounds for future work.

The maximum increase in tendon length (strain at ultimate load or $\varepsilon \_\max$ ) of $279 \% \pm 80 \%$ was found in tendons cut with a helical angle of $30^{\circ}$ and without subsequent suturing (group III). This group also had high variability in biomechanical performance, with strain at ultimate load values that ranged from $210 \%$ to $410 \%$ (Fig. 4). This can be partly explained by the observation that the cut line is longest if a $30^{\circ}$ helical angle is used, and this increases the likelihood of geometric irregularities (points of failure). Furthermore, as mentioned above, more severe helical angles may be limited by lack of effective shear transfer of loads in the tendon. Nonetheless, a $30^{\circ}$ helical cut (without sutures) yielded tendonlength increases that ranged from 5 to $15 \mathrm{~cm}$ while preserving a mean ultimate load of $30 \pm 8 \mathrm{~N}$. This represents a substantially higher tendon length compared with any full-transection technique, in which a length increase would be geometrically limited to $<5 \mathrm{~cm}$ and in which practically obtainable length increases are substantially less. It is possible that the gain in length by helical cutting could be further accentuated by disrupting the tendon at one end and partially untwisting the helix. Although disconnecting the tendon in this fashion would eliminate a key benefit of the method (preservation of continuity), untwisting could be an option for patients in whom a very large amount of additional length is needed. 
The Journal of Bone \& Joint Surgery • Jbjs. org Volume 93-A • Number 8 · April 20, 2011
Helical Cutting as a New Method for

Tendon-Lengthening in Continuity
As expected, helically cut tendons with subsequent suturing (groups IV, V, and VI) were generally stronger and substantially stiffer than tendons in the unsutured groups (groups I, II, and III) (Figs. 4 and 5). The lone exception with regard to strength was for cut angles of $30^{\circ}$, in which sutured and unsutured tendons had similar ultimate load. This indicates that severe helical angles may result in reconstructed tendons that are too fragile to effectively bear forces at the sutures.

The second hypothesis was that the length and tensile strength could be controlled by an a priori choice of helical cut angle. This was confirmed in cut tendons that were not sutured; smaller helical angles were well correlated to additional length and reduced load-bearing capacity. It should be noted that it is also possible to control the length increase by adjusting the length of the cutting zone itself. It is mandatory, however, that the cut be implemented such that it circumscribes the tendon at least once; otherwise, no lengthening (or very asymmetrical lengthening) occurs. If the helically cut tendons were sutured, strain at ultimate load did not necessarily correspond to the cut angle; however, there was nonetheless a strong positive correlation between ultimate load and the helical angle. Thus, suture retention strength seems to be critically related to the cut angle.

Suturing of the helically cut tendons was performed in these experiments with use of a nominal pretension of $5 \mathrm{~N}$ to standardize the suturing procedure. Preliminary experiments indicated that lower pretensions were insufficient to align the cut surfaces for suturing. Higher pretensions were not investigated in the current study, but they effectively lengthen the tendon prior to suturing and represent a large potential for controlling the target length of the reconstructed tendon in various surgical situations.

The third hypothesis was that helically cut tendons would tolerate significantly higher tensile strength than that tolerated by tendons lengthened with use of z-plasty. While this was the case with cutting angles of $45^{\circ}$ or higher (both sutured and not), this was not the case in groups III and VI, with the highly oblique cut angle of $30^{\circ}$. However, as mentioned above, these groups could attain extreme additional length (Fig. 4).

In conclusion, helical cutting provides significantly greater potential for tendon-lengthening than $\mathrm{z}$-plasty does. Helical lengthening at cut angles $>45^{\circ}$ also provides superior tensile strength. Furthermore, helical cutting does not fully disrupt tendon continuity, and this may have positive implications for healing. Although healing studies remain to be done, the in vitro analysis we present indicates that the method could offer technical and/or biomechanical advantages over conventional tendon-lengthening techniques.

Mazda Farshad, MD

Christian Gerber, MD, FRCSEd(Hon)

Jess G. Snedeker, PhD

Dominik C. Meyer, MD

Department of Orthopaedics,

University Hospital Balgrist,

Forchstrasse 340, 8008 Zürich, Switzerland.

E-mail address for M. Farshad: mazda.farshad@balgrist.ch

\section{References}

1. Hove CR, Williams EF 3rd, Rodgers BJ. Z-plasty: a concise review. Facial Plast Surg. 2001;17:289-94.

2. Borges AF. The enigma of Serre's "Z-plasty" technique. Plast Reconstr Surg. 1985;76:472-4.

3. Hatt RN, Lamphier TA. Triple hemisection: a simplified procedure for lengthening the Achilles tendon. N Engl J Med. 1947;236:166-9.

4. Vulpius $O$, Stoffel A. Tenotomie der Endsehnen der Mm. gastrocnemius et soleus. Orthopädische Operationslehre. Stuttgart: Ferdinand Enke; 1913. p 29-31.

5. Baker LD. A rational approach to the surgical needs of the cerebral palsy patient. J Bone Joint Surg Am. 1956;38:313-23.

6. White JW. Torsion of the Achilles tendon: its surgical significance. Arch Surg. 1943;46:784-7.

7. Clarke HD, Fuchs R, Scuderi GR, Scott WN, Insall JN. Clinical results in valgus total knee arthroplasty with the "pie crust" technique of lateral soft tissue releases. J Arthroplasty. 2005;20:1010-4.

8. Shigley JE, Mitchell LD. Mechanical engineering design. 4th ed. New York: McGraw-Hill; 1983.

9. Gideroglu K, Akan M, Orhun H, Bozdag E, Gül AE, Akgun E, Akoz T. In vivo comparison of biomechanical, histological, and radiological properties of three techniques for tendon lengthening: an experimental study in rabbits. Scand J Plast Reconstr Surg Hand Surg. 2009;43:1-7.
10. Aktas S, Ercan S, Candan L, Moralar U, Akata E. Early mobilization after sliding and Z-lengthening of heel cord: a preliminary experimental study in rabbits. Arch Orthop Trauma Surg. 2001;121:87-9.

11. Costa ML, Donell ST, Tucker $K$. The long-term outcome of tendon lengthening for chronic Achilles tendon pain. Foot Ankle Int. 2006;27:672-6.

12. Sayed-Noor AS, Pedersen E, Wretenberg $P$, Sjödén GO. Distal lengthening of ilio-tibial band by Z-plasty for treating refractory greater trochanteric pain after total hip arthroplasty (Pedersen-Noor operation). Arch Orthop Trauma Surg. 2009;129:597-602.

13. Ahmed IM, Lagopoulos M, McConnell P, Soames RW, Sefton GK. Blood supply of the Achilles tendon. J Orthop Res. 1998;16:591-6.

14. Gerber C, Meyer DC, Schneeberger AG, Hoppeler H, von Rechenberg B. Effect of tendon release and delayed repair on the structure of the muscles of the rotator cuff: an experimental study in sheep. J Bone Joint Surg Am. 2004;86:1973-82.

15. Maffulli N, Barrass V, Ewen SW. Light microscopic histology of Achilles tendon ruptures. A comparison with unruptured tendons. Am J Sports Med. 2000;28: 857-63.

16. Tallon C, Maffulli N, Ewen SW. Ruptured Achilles tendons are significantly more degenerated than tendinopathic tendons. Med Sci Sports Exerc. 2001;33:1983-90. 17. Ismail M, Karim A, Shulman R, Amis A, Calder J. The Achillon Achilles tendon repair: is it strong enough? Foot Ankle Int. 2008;29:808-13. 